\title{
Retinal Blood Flow as a Predictor of Recurrence of Macular Edema after Intravitreal Ranibizumab Injection in Central Retinal Vein Occlusion
}

\author{
Yurika Takano ${ }^{a}$ Hidetaka Noma $^{a}$ Kanako Yasuda ${ }^{a}$ Tomoe Yamaguchia \\ Hiroshi Gotob Masahiko Shimura ${ }^{a}$ \\ ${ }^{a}$ Department of Ophthalmology, Hachioji Medical Center, Tokyo Medical University, Tokyo, Japan; ${ }^{\mathrm{b}}$ Department of \\ Ophthalmology, Tokyo Medical University, Tokyo, Japan
}

\section{Keywords}

Retinal blood flow · Laser speckle flowgraphy · Macular edema $\cdot$ Recurrence $\cdot$ Central retinal vein occlusion

\begin{abstract}
Introduction: To investigate the relationship between retinal blood flow and the presence or absence of macular edema $(\mathrm{ME})$ recurrence after intravitreal ranibizumab injection (IRI) in patients with central retinal vein occlusion (CRVO). Methods: We reviewed the medical records of 16 eyes with ME associated with CRVO. All eyes had received pro re nata IRI. Repeat IRI was performed if the central macular thickness was $\geq 300 \mu \mathrm{m}$. At 12 months, patients without additional IRI in the past 6 months were assigned to the resolved group, and those with additional IRI, to the recurrence group. We used laser speckle flowgraphy (LSFG) to measure the mean blur rate (MBR) of the optic disc before and after IRI. Results: Ten of the 16 eyes were assigned to the resolved group, and the other 6 eyes to the recurrence group. At several visits in the 12 months after IRI, MBR was significantly higher in the resolved group than in the recurrence group. Percent change of MBR (\% $\triangle \mathrm{MBR}$ ) from baseline was significantly higher in the resolved group than in the recurrence group at 1 month (initial \% $\triangle \mathrm{MBR}$ ) and 11 and 12 months. Multivariate stepwise analysis showed that the initial $\% \triangle M B R$ was significantly and negatively correlated with the number of IRIs. Discus-
\end{abstract}

sion/Conclusion: These findings suggest that determining $\% \triangle M B R$ in LSFG may be a useful way to determine the likelihood of ME recurrence in CRVO patients.

(c) 2021 The Author(s).

Published by S. Karger AG, Basel

\section{Introduction}

Central retinal vein occlusion (CRVO) is common in patients with lifestyle-related diseases such as hypertension and arteriosclerosis. Macular edema (ME) occurs frequently in CRVO and is the main cause of visual impairment in this disorder. Progression of ischemia in CRVO can result in neovascular glaucoma, a relevant risk factor that causes rapid vision loss and has a poor prognosis [1].

In CRVO, the retinal central vein is compressed by an arteriosclerotic central retinal artery in the vicinity of the lamina cribrosa, leading to venous thrombosis [2]. In the acute phase of CRVO, pressure increases in the capillaries and venules affected by the obstruction, which results in breakdown of the blood-retinal barrier and leakage of blood components into the eye. In $\mathrm{ME}$, fluid accumulates in the inner to outer plexiform layer in the retina, perhaps because of an alteration of retinal blood flow [3]. CRVO leads to increased resistance to blood flow in retinal arterioles, causing closure of retinal capillaries and small ar-
C 2021 The Author(s).

Published by S. Karger AG, Basel

This is an Open Access article licensed under the Creative Commons Attribution-NonCommercial-4.0 International License (CC BY-NC) (http://www.karger.com/Services/OpenAccessLicense), applicable to the online version of the article only. Usage and distribution for commercial purposes requires written permission.
Correspondence to:

Hidetaka Noma, noma-hide@umin.ac.jp 
terioles and resulting in retinal hypoxia [4]. Thus, understanding the retinal hemodynamic abnormalities that underlie the pathogenesis of CRVO is of critical importance.

Laser speckle flowgraphy (LSFG) is a noninvasive, real-time method that can measure the relative blood flow of the optic nerve head [5, 6]. Matsumoto et al. [7] recently reported that blood flow changes after anti-vascular endothelial growth factor (VEGF) therapy, and novel findings from Cehofski et al. [8] based on proteomic studies indicate that VEGF regulates a number of proteins involved in formation of $\mathrm{ME}$ in retinal vein occlusion. Induction of VEGF by vascular occlusion was shown to be associated with ME with CRVO [9]. Clinical trials on anti-VEGF therapy have led to the approval of its use as a treatment for ME with CRVO. Several randomized clinical trials have demonstrated better visual prognosis, represented as a 12-13-letter gain from baseline, with repeated intravitreal anti-VEGF therapy for ME associated with CRVO. However, the required number of anti-VEGF injections was $12-13$ per 2-year period [1012]. Furthermore, unclear is whether retinal blood flow is involved in the recurrence of ME. Therefore, we evaluated retinal blood flow before and after anti-VEGF therapy in patients with CRVO and examined the relationship between retinal blood flow and the presence or absence of recurrence of $\mathrm{ME}$ in these patients.

\section{Materials and Methods}

\section{Subjects}

This retrospective study was conducted in accordance with the Declaration of Helsinki. After approval by the Review Committee of the Institutional Research Board of Tokyo Medical University Hachioji Medical Hospital, consecutive CRVO patients with ME were enrolled in the study and underwent intravitreal ranibizumab injection (IRI) (Lucentis; $0.5 \mathrm{mg}$ in $0.05 \mathrm{~mL}$; Genentech, Inc., South San Francisco, CA, USA) at Tokyo Medical University Hachioji Medical Hospital. After obtaining informed consent from the patients, an IRI was performed via the pars plana using a 30 -gage needle. Injections were performed at $3.5 \mathrm{~mm}$ posterior to the limbus.

Patients with CRVO who were scheduled to undergo IRI for treatment of ME between June 2015 and July 2019 were eligible for the study. CRVO was diagnosed by fluorescein angiography with a Digital Retinal Camera CF-1 (Canon, Melville, NY, USA). Criteria for receiving IRI were ME involving the fovea (central macular thickness $[\mathrm{CMT}]>300 \mu \mathrm{m}$ ) and a best-corrected visual acuity (BCVA) of $<25 / 30$. Patients were evaluated every month for 12 months, and IRI was repeated if the CMT was $\geq 300 \mu \mathrm{m}$ and after obtaining consent from the patient. At 12 months, patients without additional IRI in the past 6 months were assigned to the resolved group, and those with additional IRI in that timeframe, to the recurrence group. Patients were excluded if proper measurements could not be obtained (e.g., in case of a refractive error of $<-6.0$ diopters, corneal opacity, poor mydriasis, cataracts with severe opacity, or vitreous hemorrhage) and if they had been followed up for $<12$ months. Additional exclusion criteria were a diagnosis of ischemic CRVO, glaucoma, aphakia, rubeosis iridis, diabetes mellitus with diabetic retinopathy, or ocular infection; previous ocular inflammation, macular laser photocoagulation, intravitreal injection of anti-VEGF agents, treatment with steroids, or vitreoretinal surgery; and concomitant administration of anti-inflammatory comedication.

\section{LSFG Blood Flow Measurements}

LSFG was performed at baseline and at every visit after IRI. The mean blur rate (MBR), obtained by LSFG (LSFG-NAVI; Softcare Co., Ltd, Fukuoka, Japan), is a quantitative index of the relative blood flow velocity, as reported in detail previously $[5,6,13]$. By using offline analysis software (LSFG Analyzer, version 3.0.47.0), we combined all images and converted them to color-coded maps in which each pixel was assigned a computed MBR. The MBR was expressed in arbitrary units (AU) and displayed as a 2-dimensional, color-coded map of blood flow velocity. After manually defining a circle around the optic disc with a rubber band [14-16], we investigated the MBR within this region. We fitted an elliptical band around the optic disc, so measurements of MBR within the region were not affected by the vessel tortuousness. Therefore, we were able to measure MBR in all patients. The MBR in the optic disc area includes choroidal blood flow; therefore, to exclude any influence of choroidal blood flow on retinal blood flow in the major vessels (arteries and veins), we subtracted the mean MBR of the tissue area from that of the vascular area $[7,17]$. All measurements were performed in triplicate, and the mean MBR value was calculated. Eye positions were recorded by performing LSFG with an auto-tracking function, which enabled us to capture the same area again during subsequent examinations with high reproducibility. To evaluate changes of retinal blood flow, we calculated the percent change of $\mathrm{MBR}(\% \Delta \mathrm{MBR})$ from baseline, as follows: $\% \Delta \mathrm{MBR}=\left(\mathrm{MBR}_{\text {each }}\right.$ month $\left.-\mathrm{MBR}_{\text {baseline }}\right) / \mathrm{MBR}_{\text {baseline }} \times 100$, where $\mathrm{MBR}_{\text {baseline }}$ and $\mathrm{MB}$ -

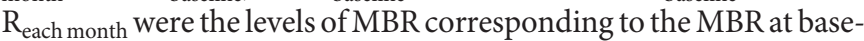
line and at each month after the initial IRI, respectively. All blood flow measurements were performed before intravitreal injection.

\section{Routine Examinations}

Each month, in addition to LSFG, all patients underwent a complete ophthalmic examination, including decimal BCVA and spectral-domain optical coherence tomography with the Spectralis imaging platform (Heidelberg Engineering, Heidelberg, Germany). At each follow-up, we determined BCVA and performed optical coherence tomography. The CMT was defined as the distance between the inner limiting membrane and the retinal pigment epithelium (including any serous retinal detachment) and was automatically measured by computer software. For statistical analysis, BCVA was converted to the logarithm of the minimal angle of resolution (log MAR) scale.

\section{Hemodynamics}

In healthy people with normal eyes, the relationship between MBR and ocular perfusion pressure (OPP) is bilinear within a certain range [18]. Therefore, to exclude physiological responses from the present results we calculated the OPP on the basis of blood pressure and intraocular pressure (IOP). First, we calculated mean blood pressure (MBP) from systolic blood pressure (SBP) and diastolic blood pressure $(\mathrm{DBP})$ as $\mathrm{MBP}=\mathrm{DBP}+1 / 3(\mathrm{SBP}-\mathrm{DBP})$. Then, we calculated OPP by using the following equation: $\mathrm{OPP}=2 / 3 \mathrm{MBP}-\mathrm{IOP}$. 
Statistical Analysis

All analyses were performed with SAS System 9.4 software (SAS Institute Inc., Cary, NC, USA). Results are presented as the mean \pm standard deviation. We compared unpaired continuous variables with an unpaired Student's $t$ test and paired continuous variables with a paired Student's $t$ test. A multivariable linear regression model with stepwise variable selection after log transformation was performed to determine the influence of the various factors. Two-tailed $p$ values of $<0.05$ were considered to indicate a significant difference.

\section{Results}

A total of 16 consecutive patients with CRVO (16 eyes) were eligible to be included in the study ( 11 men and 5 women), with a mean age of $69.3 \pm 13.8$ years. None of these patients had any previous treatments for $\mathrm{ME}$ related to CRVO. The mean duration of $\mathrm{ME}$ was $25.8 \pm 8.7$ days (range, 14-47 days). Fourteen of the 16 patients $(87.5 \%)$ had hypertension, which was defined as current treatment with antihypertensive drugs or a blood pressure $>140 / 90 \mathrm{~mm} \mathrm{Hg}$, and 5 of the 16 patients (31.3\%) had hyperlipidemia. The mean SBP was $137 \pm 9.5 \mathrm{~mm} \mathrm{Hg}$; mean DBP, $85 \pm 9.1 \mathrm{~mm} \mathrm{Hg}$; MBP, $102 \pm 7.7 \mathrm{~mm} \mathrm{Hg}$; and OPP, $87 \pm 7.6 \mathrm{~mm} \mathrm{Hg}$. The mean baseline BCVA was logMAR $0.60 \pm 0.49$; mean baseline CMT, $811 \pm 260 \mu \mathrm{m}$; and mean baseline MBR, $18.8 \pm 5.95$ AU. Both BCVA (logMAR $0.20 \pm 0.37)$ and CMT $(253 \pm 64.7 \mu \mathrm{m})$ improved significantly at 12 months after initial IRI in all eyes $(p<0.001$ and $p<0.001$, respectively), but the mean MBR was not significantly different between baseline and 12 months $(21.1 \pm 8.69 \mathrm{AU} ; p=0.137)$. In addition, we found no cases of conversion from the nonischemic to the ischemic type of CRVO during the 12 months.

At 12 months after initial IRI, 10 of the 16 eyes (62.5\%) with ME associated with CRVO had required no additional IRI in the past 6 months. These 10 eyes were assigned to the resolved group, and the other 6 eyes (37.5\%), to the recurrence group. The clinical characteristics of the 2 groups are summarized in Table 1. We found no significant differences in the sex distribution or baseline values of any clinical parameters (duration of $\mathrm{ME}$, hypertension, SBP, DBP, hyperlipidemia, BCVA, CMT, MAP, $\mathrm{OPP}$, and MBR) between the resolved and recurrence groups, but age was significantly different between the 2 groups $(p=0.012)$ (Table 1$)$.

BCVA improved significantly more in the resolved group than in the recurrence group at each month (3 months, ${ }^{*} p=0.008 ; 5$ months, ${ }^{*} p=0.036 ; 6$ months, ${ }^{*} p=$ $0.007 ; 7$ months, ${ }^{*} p=0.021 ; 9$ months, ${ }^{*} p=0.049 ; 10$ months, ${ }^{*} p=0.025 ; 11$ months, ${ }^{*} p=0.009$; and 12 months,
Table 1. Baseline clinical features of the resolved and recurrence groups

\begin{tabular}{llll}
\hline Findings & $\begin{array}{l}\text { Resolved } \\
\text { group }(N=10)\end{array}$ & $\begin{array}{l}\text { Recurrence } \\
\text { group }(N=6)\end{array}$ & $\begin{array}{l}p \\
\text { value }\end{array}$ \\
\hline Age, years & $62.7 \pm 14.4^{\ddagger}$ & $80.1 \pm 2.3^{\ddagger}$ & 0.012 \\
Gender (female/male) & $2 / 8$ & $3 / 3$ & 0.210 \\
Duration of ME, days & $25.5 \pm 7.27^{\ddagger}$ & $26.2 \pm 12.1^{\ddagger}$ & 0.892 \\
Hypertension & 8 & 6 & 0.242 \\
SBP, mm Hg & $135 \pm 11^{\ddagger}$ & $140 \pm 7.6^{\ddagger}$ & 0.345 \\
DBP, mm Hg & $87 \pm 7.4^{\ddagger}$ & $80 \pm 11^{\ddagger}$ & 0.130 \\
Hyperlipidemia & 3 & 2 & 0.889 \\
Baseline BCVA (logMAR) & $0.50 \pm 0.50^{\ddagger}$ & $0.77 \pm 0.48^{\ddagger}$ & 0.316 \\
Baseline CMT, um & $730 \pm 167^{\ddagger}$ & $945 \pm 342^{\ddagger}$ & 0.111 \\
MBP, mm Hg & $103 \pm 6.9^{\ddagger}$ & $100 \pm 9.7^{\ddagger}$ & 0.443 \\
OPP, mm Hg & $89 \pm 6.1^{\ddagger}$ & $85 \pm 10^{\ddagger}$ & 0.374 \\
Baseline MBR, AU & $19.8 \pm 5.51^{\ddagger}$ & $17.2 \pm 6.83^{\ddagger}$ & 0.417 \\
\hline
\end{tabular}

BCVA, best-corrected visual acuity; CMT, central macular thickness; logMAR, logarithm of the minimum angle of resolution; $\mathrm{MBP}$, mean blood pressure; MBR, mean blur rate; OPP, ocular perfusion pressure; $M E$, macular edema; $A U$, arbitrary units; $S B P$, systolic blood pressure; DBP, diastolic blood pressure. ${ }^{\ddagger}$ Mean \pm standard deviation.

$\left.{ }^{*} p=0.023\right)$ (Fig. 1a). CMT decreased significantly more in the resolved group than in the recurrence group at 4 months $\left({ }^{*} p=0.047\right)$ and 6 months $(* p=0.002)$ (Fig. $\left.1 b\right)$.

The MBR was significantly higher in the resolved group than in the recurrence group at each month (1 month, ${ }^{*} p=0.013 ; 2$ months, ${ }^{*} p=0.017 ; 3$ months, ${ }^{*} p=$ $0.038 ; 4$ months, ${ }^{*} p=0.018 ; 8$ months, ${ }^{*} p=0.022 ; 9$ months, ${ }^{*} p=0.025 ; 10$ months, ${ }^{*} p=0.030 ; 11$ months, ${ }^{*} p=0.017$; and 12 months, ${ }^{*} p=0.004$ ) (Fig. 2a). The $\% \Delta$ MBR from baseline was significantly higher in the resolved group than in the recurrence group at 1 month (initial $\% \Delta \mathrm{MBR})\left({ }^{*} p=0.010\right), 11$ months $\left({ }^{*} p=0.017\right)$, and 12 months $\left({ }^{*} p<0.001\right)$ (Fig. $\left.2 b\right)$.

At 12 months, the number of times IRI had been performed was as follows: once, 4 eyes; twice, 5 eyes; 3 times, 2 eyes; 4 times, 2 eyes; and 5 or more times, 3 eyes. The mean number of IRIs was significantly higher in the recurrence group than in the resolved group $(5.0 \pm 1.5$ vs. $1.7 \pm 0.67$, respectively; $p<0.001$ ). Table 2 shows the associations of the number of IRIs with clinical characteristics. In all eyes and patients, the initial $\% \triangle \mathrm{MBR}$ was significantly and negatively correlated with the number of IRIs and age was significantly correlated with the number of IRIs. Only the initial $\% \Delta \mathrm{MBR}$ was identified as a significant factor in the stepwise multivariable analysis. 
Fig. 1. BCVA and central macular thickness after IRI. a BCVA improved more in the resolved group (solid line) than in the recurrence group (dotted line) at several visits (baseline, $p=0.316 ; 1$ month, $p=$ $0.168 ; 2$ months, $p=0.107 ; 3$ months, ${ }^{*} p=$ $0.008 ; 4$ months, $p=0.061 ; 5$ months, ${ }^{*} p=$ $0.036 ; 6$ months, ${ }^{*} p=0.007 ; 7$ months, ${ }^{*} p=$ $0.021 ; 8$ months, $p=0.067 ; 9$ months, ${ }^{*} p=$ $0.049 ; 10$ months, ${ }^{*} p=0.025 ; 11$ months, ${ }^{*} p=0.009$; and 12 months, ${ }^{*} p=0.023$ ). b Central macular thickness showed a significant difference between the resolved group (solid line) and recurrence group (dotted line) only at 4 and 7 months (baseline, $p=0.111 ; 1$ month, $p=0.273$; 2 months, $p=0.090 ; 3$ months, $p=0.206$; 4 months, ${ }^{*} p=0.047 ; 5$ months, $p=0.368$; 6 months, ${ }^{*} p=0.002$; 7 months, $p=0.205$; 8 months, $p=0.053$; 9 months, $p=0.129$; 10 months, $p=0.065 ; 11$ months, $p=0.054$; and 12 months, $p=0.455)$. BCVA $\log$ MAR, best-corrected visual acuity converted to the logarithm of minimal angle of resolution; CMT, central macular thickness; IRI, intravitreal ranibizumab injection.

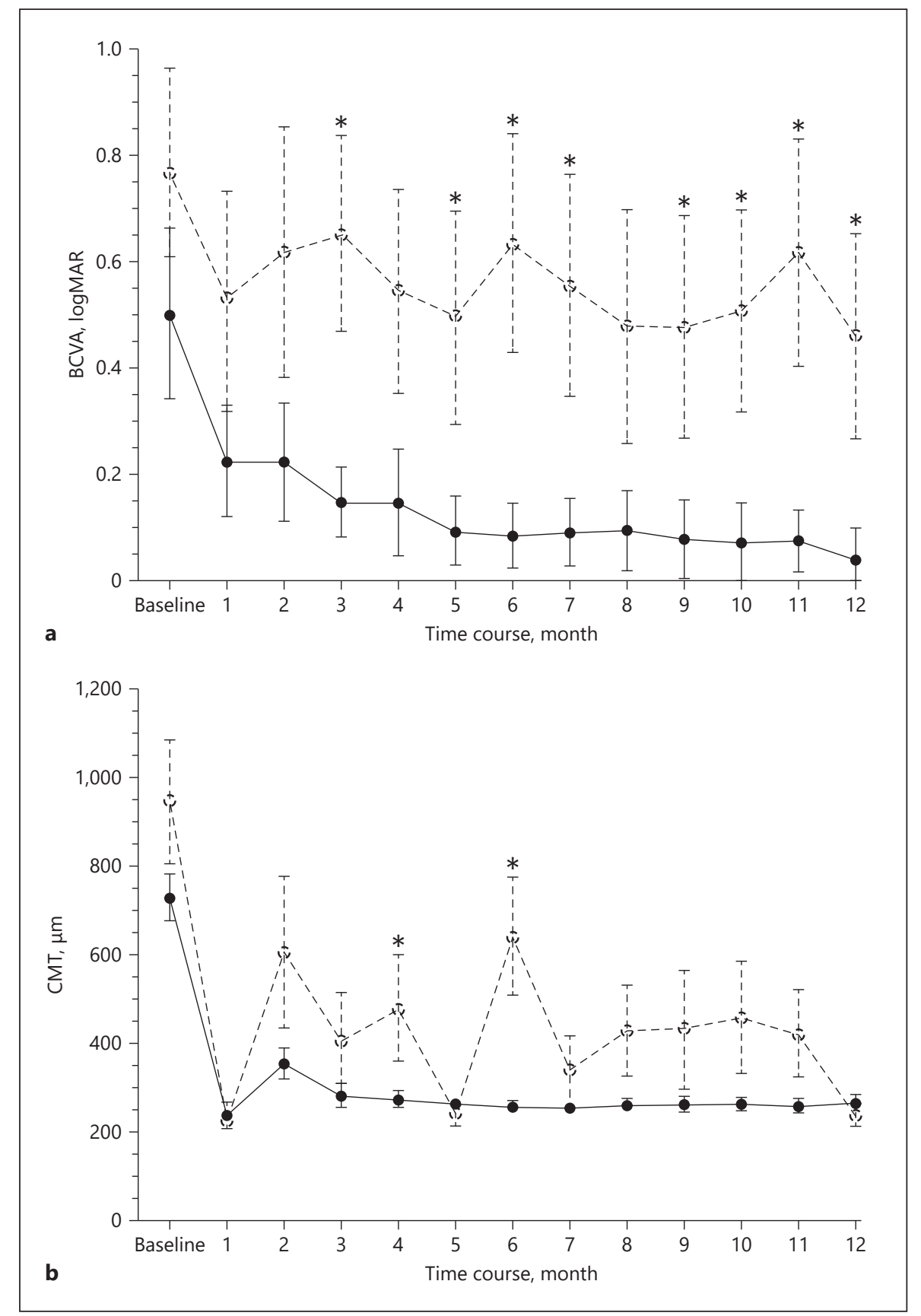

\section{Discussion/Conclusion}

In this study, we evaluated whether retinal blood flow is involved in the recurrence of ME by measuring retinal blood flow before and after anti-VEGF therapy in patients with CRVO and examining the relationship between retinal blood flow and the presence or absence of recurrence of $\mathrm{ME}$ in these patients. The percent change of MBR ( $\% \Delta \mathrm{MBR})$ from baseline was significantly higher in the resolved group than in the recurrence group at 1 month (initial $\% \triangle \mathrm{MBR}$ ) and 11 and 12 months. In addition, multivariate analysis showed that the initial \% $\triangle \mathrm{MBR}$ was significantly and negatively correlated with the number of IRIs during the 12 -month study period. These findings suggest that the better the initial improvement in retinal blood flow after antiVEGF therapy, the less likely recurrence of ME is.

Previously, development of ME in patients with CRVO was hypothesized to be caused by movement of fluid from 
Fig. 2. MBR and percent change of MBR after IRI. a MBR was significantly higher in the resolved group (solid line) than in those in the recurrence group (dotted line) at several visits (baseline, $p=0.417 ; 1$ month, ${ }^{*} p=0.013 ; 2$ months, ${ }^{*} p=0.017 ; 3$ months, ${ }^{*} p=0.038 ; 4$ months, ${ }^{*} p=0.018 ; 5$ months, $p=0.108 ; 6$ months, $p=0.241 ; 7$ months, $p=0.074 ; 8$ months, ${ }^{*} p=0.022 ; 9$ months, ${ }^{*} p=0.025 ; 10$ months, ${ }^{*} p=0.030 ; 11$ months, ${ }^{*} p=0.017$; and 12 months, ${ }^{*} p=$ $0.004)$. b Percent change in MBR was significantly higher in the resolved group (solid line) than in the recurrence group (dotted line) at 1 month and 11 and 12 months ( 1 month, ${ }^{*} p=0.010 ; 2$ months, $p=0.081$; 3 months, $p=0.194 ; 4$ months, $p=0.051$; 5 months, $p=0.148$; 6 months, $p=0.480$; 7 months, $p=0.118$; 8 months, $p=0.089$; 9 months, $p=0.077 ; 10$ months, $p=0.264$; 11 months, ${ }^{*} p=0.017$; and 12 months, $\left.{ }^{*} p<0.001\right)$. MBR, mean blur rate; $\% \Delta$ MBR, percent change of MBR; IRI, intravitreal ranibizumab injection.
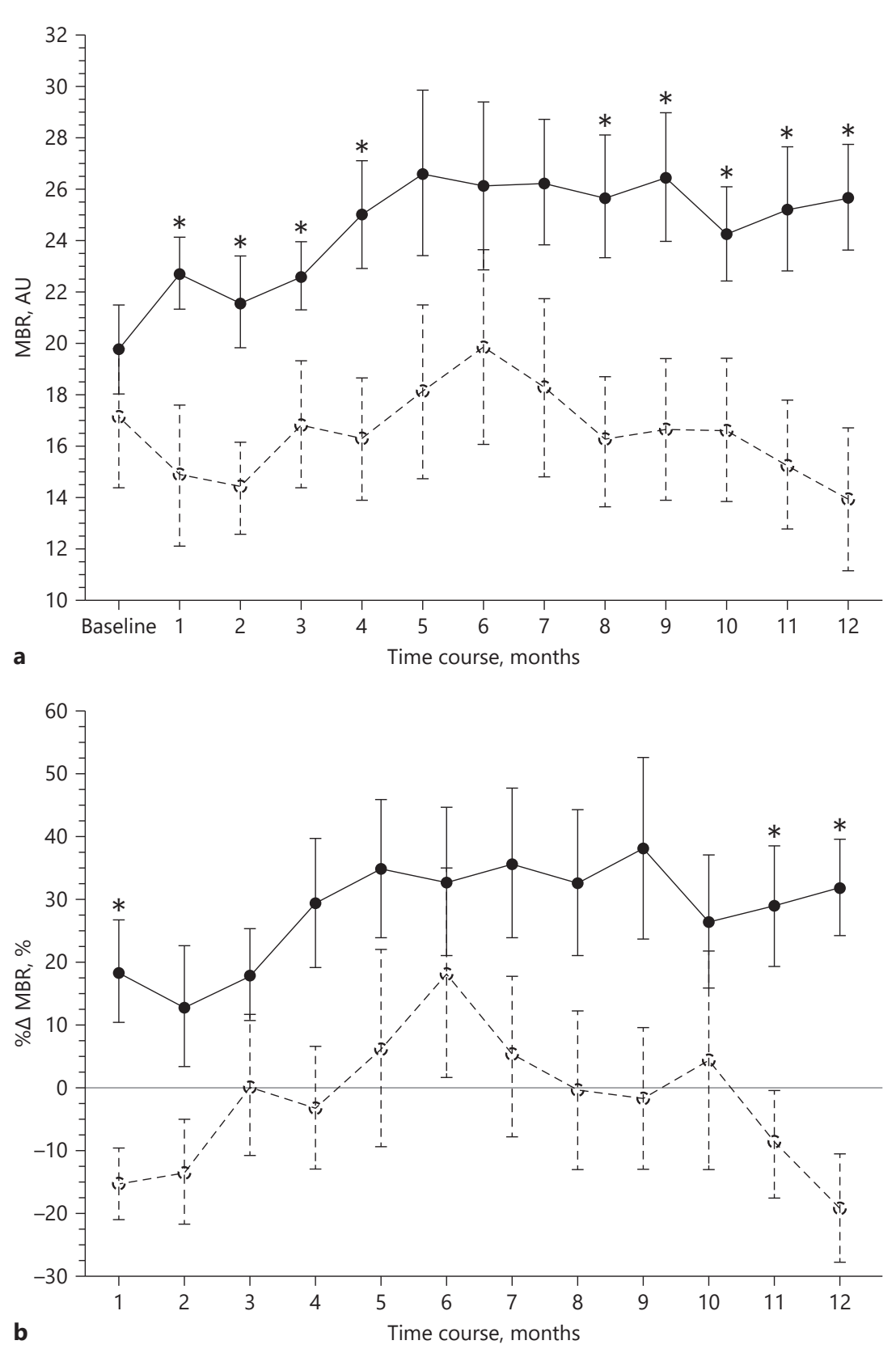

the vascular compartment to the tissues (Starling's Law) after breakdown of the blood-retinal barrier $[19,20]$. This hypothesis is supported by the finding that a decrease of retinal blood flow may lead to stagnation of retinal blood flow and subsequent recurrence of ME [21]. Taken together with the present findings, such reports confirm that recurrence of ME after IRI might be due to a decrease or stagnation of retinal blood flow. In addition, higher MBR indicates lower vascular resistance and better com- pensation, which would lead to less retinal ischemia and hypoxia. Therefore, a change of MBR, as measured by LSFG, could be an indicator of recurrence of ME.

We found that age was significantly different between the 2 groups and was significantly correlated with the number of IRIs. Advanced age is a known risk factor for CRVO [22, 23], and age also was shown to be a risk factor for CRVO among those receiving bevacizumab therapy [7]. Because vascular endothelial cell damage becomes 
Table 2. Linear regression analysis of factors related to number of IRIs

\begin{tabular}{|c|c|c|c|c|}
\hline \multirow[t]{2}{*}{ Variable } & \multicolumn{2}{|l|}{ Univariate } & \multicolumn{2}{|c|}{ Multivariate stepwise } \\
\hline & $\begin{array}{l}\text { correlation } \\
\text { coefficient }\end{array}$ & $p$ value & $\begin{array}{l}\text { correlation } \\
\text { coefficient }\end{array}$ & $p$ value \\
\hline Age, years & 0.50 & 0.048 & & \\
\hline Duration of ME, days & -0.21 & 0.427 & & \\
\hline Baseline BCVA, logMAR & 0.32 & 0.221 & & \\
\hline Baseline CMT, $\mu \mathrm{m}$ & 0.44 & 0.088 & & \\
\hline Baseline MBR, AU & -0.13 & 0.624 & & \\
\hline $\begin{array}{l}\% \triangle M B R, \% \text { (1 month from } \\
\text { baseline) }\end{array}$ & -0.63 & 0.009 & -0.63 & 0.009 \\
\hline
\end{tabular}

BCVA, best-corrected visual acuity; CMT, central macular thickness; logMAR, logarithm of the minimum angle of resolution; $M B R$, mean blur rate; $M E$, macular edema; $A U$, arbitrary units; IRI, intravitreal ranibizumab injection. more severe in older age [24], ME might be more likely to recur because of damage to tight junctions [25]. These results suggest that older patients with CRVO have a higher risk of recurrence of ME, and they are supported by our finding that recurrence of ME was significantly associated with age. Thus, age might be a useful indicator of necessary treatment intensity.

We also found that the final visual acuity was significantly different between the resolved and recurrence groups, although we found no significant difference in CMT between the groups at the final visit. Earlier studies showed that visual acuity is strongly correlated with the integrity of the photoreceptors [26, 27]. In CRVO, each recurrence of ME could cause further damage to photoreceptors, which might explain why the final visual acuity was significantly different between the 2 groups. In the case of older patients or lack of improvement of blood flow after anti-VEGF therapy, switching to another treatment may be the best approach because of the possibility of recurrence of $\mathrm{ME}$.

The present study was limited by the small sample size, which meant that we compared only 10 patients in the resolved group with 6 patients in the recurrence group. Nevertheless, we found that change in retinal blood flow may influence the number of IRIs required in eyes with CRVO. Further studies in larger samples are required to clarify the relationship between retinal blood flow and recurrence of ME. If confirmed, our results may indicate that LSFG could potentially save some follow-up visits for some patients with CRVO because high MBR may predict a favorable outcome. In addition, the clinical pictures of nonischemic and ischemic CRVO are generally quite different [28], which is why we excluded patients with ischemic CRVO from this study. Consequently, patients with neovascular glaucoma, panretinal photocoagulation, and severe loss of visual acuity were also excluded. Further studies are required to compare retinal blood flow and recurrence of $\mathrm{ME}$ in nonischemic and ischemic CRVO.

In conclusion, we found that the $\% \triangle \mathrm{MBR}$ from baseline was significantly higher in the resolved group than in the recurrence group at 1 month (initial \% $\triangle \mathrm{MBR}$ ) and 11 and 12 months. The initial $\% \triangle$ MBR significantly and negatively correlated with the number of IRIs for ME associated with CRVO. Therefore, determining the percent change of MBR when measuring LSFG may be a useful way to determine the likelihood of recurrence of $\mathrm{ME}$ in eyes with CRVO.

\section{Acknowledgment}

We thank Katsunori Shimada (Department of Biostatistics, STATZ Corporation, Tokyo, Japan) for assistance with the statistical analysis.

\section{Statement of Ethics}

This study was conducted at the Department of Ophthalmology of Tokyo Medical University Hachioji Medical Center and approval was obtained from Institutional Review Board of Tokyo Medical University Hachioji Medical Center (IRB No. H-132). The procedures of the study conformed to the tenets of the Declaration of Helsinki and all patients gave written informed consent before enrollment.

\section{Conflict of Interest Statement}

No conflicting relationship exists for any author.
Takano/Noma/Yasuda/Yamaguchi/Goto/ Shimura 


\section{Funding Sources}

None. manuscript was done by Y.T. and H.N., and review and approval of the manuscript was performed by H.G. and M.S. All authors have read and approved the final manuscript.

\section{Author Contributions}

H.N. and M.S. were involved in the design and conduct of the study. Collection and management of the data were done by Y.T., T.Y., and H.N., while analysis and interpretation of the data were performed by K.Y. and M.S. Preparation of the first draft of the

\section{Data Availability Statement}

The data that support the findings of this study are not publicly available because they contain information that could compromise the privacy of research participants and because ethics committee approval for release of the data was not obtained.

\section{References}

1 Noma H, Yasuda K, Shimura M. Cytokines and pathogenesis of central retinal vein occlusion. J Clin Med. 2020;9(11):3457.

2 Hayreh SS. So-called "central retinal vein occlusion." I. Pathogenesis, terminology, clinical features. Ophthalmologica. 1976; 172(1):1-13.

3 Coscas G, Cunha-Vaz J, Soubrane G. Macular edema: definition and basic concepts. Dev Ophthalmol. 2017;58:1-10.

4 Campochiaro PA, Akhlaq A. Sustained suppression of VEGF for treatment of retinal/choroidal vascular diseases. Prog Retin Eye Res. 2021;83:100921.

5 Tamaki Y, Araie M, Kawamoto E, Eguchi S, Fujii H. Non-contact, two-dimensional measurement of tissue circulation in choroid and optic nerve head using laser speckle phenomenon. Exp Eye Res. 1995; 60(4):373-83.

6 Sugiyama T, Araie M, Riva CE, Schmetterer L, Orgul S. Use of laser speckle flowgraphy in ocular blood flow research. Acta Ophthalmol. 2010;88(7):723-9.

7 Matsumoto M, Suzuma K, Yamada Y, Tsuiki E, Fujikawa A, Kitaoka T. Retinal blood flow after intravitreal bevacizumab is a predictive factor for outcomes of macular edema associated with central retinal vein occulation. Retina. 2018;38(2):283-91.

8 Cehofski LJ, Kojima K, Terao N, Kitazawa $\mathrm{K}$, Thineshkumar S, Grauslund J, et al. Aqueous fibronectin correlates with severity of macular edema and visual acuity in patients with branch retinal vein occlusion: a Proteome Study. Invest Ophthalmol Vis Sci. 2020;61(14):6.

9 Noma H, Funatsu H, Mimura T, Harino S, Hori S. Vitreous levels of interleukin-6 and vascular endothelial growth factor in macular edema with central retinal vein occlusion. Ophthalmology. 2009;116(1):87-93.

10 Heier JS, Campochiaro PA, Yau L, Li Z, Saroj N, Rubio RG, et al. Ranibizumab for macular edema due to retinal vein occlusions: long-term follow-up in the HORIZON trial. Ophthalmology. 2012;119(4): 802-9.
11 Heier JS, Clark WL, Boyer DS, Brown DM, Vitti R, Berliner AJ, et al. Intravitreal aflibercept injection for macular edema due to central retinal vein occlusion: twoyear results from the COPERNICUS study. Ophthalmology. 2014;121(7):1414-20.

12 Larsen $M$, Waldstein SM, Priglinger S, Hykin P, Barnes E, Gekkieva M, et al. Sustained benefits from ranibizumab for central retinal vein occlusion with macular edema: 24-month results of the CRYSTAL Study. Ophthalmol Retina. 2018;2(2):13442.

13 Noma H, Yasuda K, Mimura T, Ofusa A, Shimura M. Relationship between retinal blood flow and cytokines in central retinal vein occlusion. BMC Ophthalmol. 2020; 20(1):215.

14 Chiba N, Omodaka K, Yokoyama Y, Aizawa N, Tsuda S, Yasuda M, et al. Association between optic nerve blood flow and objective examinations in glaucoma patients with generalized enlargement disc type. Clin Ophthalmol. 2011;5:1549-56.

15 Ubuka M, Sugiyama T, Onoda Y, Shiba T, Hori Y, Maeno T. Changes in the blood flow of the optic nerve head induced by different concentrations of epinephrine in intravitreal infusion during vitreous surgery. Invest Ophthalmol Vis Sci. 2014;55(3): 1625-9.

16 Yamada Y, Suzuma K, Matsumoto M, Tsuiki E, Fujikawa A, Harada T, et al. Retinal blood flow correlates to aqueous vascular endothelial growth factor in central retinal vein occulation. Retina. 2015;35(10):203742.

17 Mizui T, Noma H, Yasuda K, Kanemaki T, Goto $\mathrm{H}$, Shimura $\mathrm{M}$. Intravitreal ranibizumab reduced ocular blood flow and aqueous cytokine levels and improved retinal morphology in patients with diabetic macular edema. Sci Rep. 2020;10(1):21713.

18 Riva CE, Titze P, Hero M, Petrig BL. Effect of acute decreases of perfusion pressure on choroidal blood flow in humans. Invest Ophthalmol Vis Sci. 1997;38(9):1752-60.
19 Arnarsson A, Stefánsson E. Laser treatment and the mechanism of edema reduction in branch retinal vein occlusion. Invest Ophthalmol Vis Sci. 2000;41(3):877-9.

20 Stefansson E. The therapeutic effects of retinal laser treatment and vitrectomy. A theory based on oxygen and vascular physiology. Acta Ophthalmol Scand. 2001;79(5): 435-40.

21 Spaide RF. Retinal vascular cystoid macular edema: review and new theory. Retina. 2016;36(10): 1823-42.

22 Hayreh SS, Zimmerman MB, Podhajsky P. Incidence of various types of retinal vein occlusion and their recurrence and demographic characteristics. Am J Ophthalmol. 1994;117(4):429-41.

23 Klein R, Moss SE, Meuer SM, Klein BE. The 15-year cumulative incidence of retinal vein occlusion: the Beaver Dam Eye Study. Arch Ophthalmol. 2008;126(4):513-8.

24 Jiang F, Xu XR, Li WM, Xia K, Wang LF, Yang XC. Monotropein alleviates $\mathrm{H} 2 \mathrm{O} 2-$ induced inflammation, oxidative stress and apoptosis via NF- $\kappa \mathrm{B} / \mathrm{AP}-1$ signaling. Mol Med Rep. 2020;22(6):4828-36.

25 Yang JM, Park CS, Kim SH, Noh TW, Kim JH, Park S, et al. Dll4 suppresses transcytosis for arterial blood-retinal barrier homeostasis. Circ Res. 2020;126(6):767-83.

26 Ota M, Tsujikawa A, Murakami T, Kita M, Miyamoto K, Sakamoto A, et al. Association between integrity of foveal photoreceptor layer and visual acuity in branch retinal vein occlusion. Br J Ophthalmol. 2007; 91(12):1644-9.

27 Hasegawa T, Ueda T, Okamoto M, Ogata $\mathrm{N}$. Presence of foveal bulge in optical coherence tomographic images in eyes with macular edema associated with branch retinal vein occlusion. Am J Ophthalmol. 2014;157(2):390-6.

28 Hayreh SS, Rojas P, Podhajsky P, Montague $\mathrm{P}$, Woolson RF. Ocular neovascularization with retinal vascular occlusion-III. Incidence of ocular neovascularization with retinal vein occlusion. Ophthalmology. 1983;90(5):488-506. 